\section{Bundesforschungsbericht: Wechselwirkungen}

In ihrem achten „Bundesbericht Forschung 1988“ (BF VIII), den sie am 22. März verabschiedete, stellt die Bundesregierung zur Orientierung und als Perspektiven ihrer Forschungspolitik die immer enger werdende Verflechtung zwischen den Forschungsdisziplinen sowie zwischen Grundlagen- und angewandter Forschung, außerdem die „strukturelle“ Auswirkung der Schlüsseltechnologien auf viele Branchen heraus. Ein Versuch, den Leistungsstand von Forschung und technologischer Innovation in der Bundesrepublik darzustellen, weist auf einige bedenkliche Schwächen hin. Nach einem sehr ausführlichen allgemeinen Teil gibt der Bericht Auskunft über die Ressourcen für Wissenschaft sowie für Forschung und Entwicklung (FuE), die Schwerpunkte der FuE-Förderung des Bundes, die Forschungsförderung in den Bundesländern, die internationale und innerdeutsche Zusammenarbeit sowie die Förderorganisationen und Forschungseinrichtungen in der Bundesrepublik. Der Statistikteil enthält 41 Tabellen zu Forschungsaufwendungen und -personal unter unterschiedlichen Aspekten.

Der BF VIII ist von dem Bemühen gekennzeichnet, die Konsolidierung der von der konservativ-liberalen Regierung 1982 eingeleiteten Forschungspolitik zu dokumentieren und - in Grenzen - neue Akzente zu setzen. Als Schwerpunkte werden herausgestellt: Grundlagenforschung, Vorsorgeforschung, stärkere Förderung von Schlüsseltechnologien bei gleichzeitig zurückgehender Förderung marktnaher Projekte in der Wirtschaft, Alternativen für Klein- und Mittelunternehmen zum Wegfall der meisten indirekten (Personal-) Förderung, Langfristprogramme und internationale Kooperation.

Der Leistungsstand von Forschung und Technologie ist in der Bundesrepublik schwer festzustellen. Den Rückstand der Wissenschaftsforschung in diesem Punkt gegenüber anderen Industrieländern will das Bundesforschungsministerium (BMFT) vermindern helfen mit der Vergabe von Studien und indem es die Methodenentwicklung unterstützt. Der wissenschaftliche Wettbewerb soll ermuntert werden. Nach der Zahl der natur- und ingenieurwissenschaftlichen Publikationen nimmt die Bundesrepublik den vierten Platz hinter den USA, Japan und Großbritannien ein, allerdings mit weit geringerer Konzentration auf die Schlüsselbereiche Biotechnologie, Mikroelektronik, Informationstechnik und Materialwissenschaften. In klassischen naturwissenschaftlichen Bereichen, insbesondere in der Physik, ist die Bundesrepublik stark. Die im Vergleich mit anderen Ländern „etwas geringere Dynamik beim Aufgreifen neuer Wissensgebiete" entspricht laut BF VIII „den Aussagen der Wirtschaft über Defizite in der anwendungsorientierten Grundlagenforschung“. Der Regierungsbericht bestätigt, was die Deutsche Forschungsgemeinschaft in ihrem mittelfristigen Plan zur
Notwendigkeit interdisziplinärer Zusammenarbeit und $\mathrm{zu}$ den hier bestehenden Mängeln gesagt hat. Daraus ergibt sich aber auch, „daß sich der Weltmarkt vor allem bei den forschungsintensiven Waren insgesamt dynamischer entwickelt hat als der entsprechende Zugewinn deutscher forschungsintensiver Waren ausgefallen ist" - eine Erkenntnis, die in den Strukturberichten der wirtschaftswissenschaftlichen Institute vermittelt wurde.

Vor diesem Hintergrund ist wichtig, was der Bundesforschungsbericht als generellen Trend festgestellt hat: Die Grundlagenforschung insbesondere an Hochschulen orientiert sich neu. Bundesforschungsminister Riesenhuber stellt in einigen bisher als anwendungsfern angesehenen Grundlagendisziplinen, etwa Biologie und Mathematik, „ein neues Verständnis der Hochschulen zur Kooperation mit der Wirtschaft, aber auch zur außeruniversitären Forschung" fest. Sie könnten dabei im wissenschaftlichen Wettbewerb ein attraktives Profil erwerben. Er hilft kräftig mit, indem er die Verbundforschung bevorzugt fördert. In solchen Projekten bearbeiten Forscher in Hochschulen und anderen Forschungseinrichtungen zusammen mit Kollegen in Wirtschaftsunternehmen Themen, die vor allem in die Förderprogramme des BMFT passen. Die Mittel des BMFT für die Hochschulen sind von 310 Mio Mark 1982 auf 540 Mio Mark 1987 gestiegen.

Für die nächsten Jahre sieht die Bundesregierung „eine vorrangige Aufgabe darin, auf Zeit angelegte, programmorientierte Grundlagenforschung noch besser mit der freien, grundfinanzierten Forschung zu verzahnen, damit Synergieund Anstoßeffekte für beide Seiten wirksam werden“. Weniger direkt wird der Forschungspolitik die Aufgabe zugeschrieben, nicht nur die Freiheit der Forschung zu wahren, sondern „Probleme der Gesellschaft als Fragen an die Wissenschaft zu formulieren" sowie die Voraussetzungen für interdisziplinäre Teams und Verbundforschung zu schaffen. Die Kooperation mit der Wirtschaft beschneide dann die Forschungsfreiheit nicht, wenn die Wissenschaftler sich frei dafür entscheiden und „aufgrund einer gesicherten Grundausstattung und eigener hervorragender Leistungen" gleichberechtigte Partner sein könnten.

Für Forschung an den Hochschulen wurde 1987 mit 7110 Mio Mark ein Achtel aller Forschungsausgaben in der Bundesrepublik aufgewendet, für Forschung an staatlichen und privaten Instituten 13,5\% und an Einrichtungen der Wirtschaft 70,8\%. Die 56860 Mio Mark des Gesamtbudgets Forschung 1987 (45\% mehr als 1981) wurden finanziert vom Bund mit 13810 Mio Mark (24,3\% gegenüber 26,3 \% im Jahr 1981), von den Ländern mit 7600 Mio Mark $(13,4 \%$ gegenüber $16,2 \%$ ), der Wirtschaft mit 34700 Mio Mark $(61,0 \%$ gegenüber $56,1 \%)$, privaten Institutionen ohne Erwerbszweck, z.B. Stiftungen, mit 150 Mio Mark $(0,3 \%$ gegenüber $0,4 \%$ ) und vom Ausland mit 600 Mio Mark $(2,8 \%$ gegenüber 2,5\%). Die Hochschulforschung wird heute $\mathrm{zu}$ zwei Dritteln aus der Grundausstattung und zu einem Drittel 
aus Drittmitteln finanziert, davon $\mathbf{4 0 \%}$ öffentliche Mittel über die Deutsche Forschungsgemeinschaft, $30 \%$ andere öffentliche Mittel, $21 \%$ von der Wirtschaft und $9 \%$ von Stiftungen. Das Gewicht der mit Drittmitteln finanzierten Forschung wird noch zunehmen.

Der Anteil der Mittel für Grundlagenforschung an den 7576 Mio Mark des BMFT-Haushalts 1987 betrug 37\% (gegenüber $28 \%$ 1981) und soll in den nächsten Jahren auf dieser Höhe bleiben. Ein zweiter Schwerpunkt ist die Vorsorgeforschung: unter anderem rund 300 Mio Mark für Gesundheits-, ökologische, Umwelt- und Klimaforschung (75\% mehr als 1982). Für 1989 kündigt der Bericht ein Programm „neue Technologien und Arbeitsbedingungen, Arbeitsorganisation und Gesundheit am Arbeitsplatz" an. Die direkte Förderung marktorientierter Technologien in der Wirtschaft hat das BMFT seit 1982 um rund eine Milliarde Mark auf 3039 Mio Mark 1988 zurückgefahren. Für Schlüsseltechnologien jedoch, die "strukturell“ quer durch viele Wirtschaftsbereiche wirken, sind die Mittel um 35\% erhöht worden. Dazu zählen Informationstechnik, Materialforschung, Biotechnologie, Airbus-Entwicklung sowie ausgewählte physikalische Technologien wie Laser- und Supraleitungstechnik. Nachdem die wichtigsten Programme der indirekten Förderung von Forschungspersonal insbesondere an kleinen und mittleren Unternehmen weggefallen sind, überlegt man sich im BMFT neue Wege. Die „Forschungskooperation zwischen Industrie und Wissenschaft" soll weitergeführt, Demonstrationslabors und die industrielle Gemeinschaftsforschung sollen ausgebaut werden. Forschungsgebiete mit langfristiger und internationaler Perspektive wie Kernfusions-, Meeres- und Polar- sowie Weltraumforschung will die Bundesregierung stärken. Auch solle sich die Bundesrepublik zunehmend in der internationalen Zusammenarbeit engagieren.

\section{FuE-Output in der Patentstatistik}

Mit einem neuen Analyseinstrument können jetzt die $\mathrm{Zu}$ sammenhänge zwischen Forschung und Entwicklung einerseits und der industriellen Innovation andererseits deutlich gemacht werden. Für die SV-Wissenschaftsstatistik GmbH, ein Tochterunternehmen des Stifterverbandes für die Deutsche Wissenschaft, haben Dr. S. Greif (Deutsches Patentamt) und G. Potkowik (vormals Statistisches Bundesamt) eine Studie „Zusammenführung der Internationalen $\mathrm{Pa}$ tentklassifikation und der Systematik der Wirtschaftszweige" erarbeitet. Der Vorsitzende des Wissenschaftlichen Beirats der SV-Wissenschaftsstatistik, Prof. H. Albach (Berlin/ Koblenz), hat sie am 21. März in Bonn vorgestellt. Mit Hilfe dieses Konkordanzsystems können technische und wirtschaftliche Sachverhalte einander zugeordnet, Wirtschaftsmit Patentstatistiken verknüpft werden. Es gibt Auskunft darüber, aus welchen Wirtschaftszweigen die Erfindungen kommen und in welchen sie angewendet werden.

Während die FuE-Statistik bisher nur den Input erfaßte, damit aber der Dynamik der Entwicklung nicht gerecht werden konnte, kann man nun an Hand von Patentdaten den Output von FuE ermitteln. In den Patentdatenbanken PATDPA, PATOS und INPADOC sowie im Patentblatt und der
Patentrolle des Deutschen Patentamtes wurden 26060 erstveröffentlichte inländische Patentanmeldungen aus dem Jahre 1983 festgestellt. Die Studie basiert auf der Untersuchung der Patente derjenigen, die mindestens zehn angemeldet hatten - insgesamt 12990 Anmeldungen von 279 Anmeldern. Noch umfangreichere Zuordnungsarbeiten sind notwendig, wenn man das Ziel erreichen will, „Technologien von einer frühen Phase der Entstehung an bis zur Anwendung hin $\mathrm{zu}$ verfolgen und Richtung, Umfang, Geschwindigkeit und Streuung von Technologieeinflüssen zu erfassen“. Es sei möglich, so der Bericht des Präsidenten des Deutschen Patentamtes, Dr. E. Häußer, Aktivitäten in eng begrenzten wirtschaftlichen und technischen Gebieten, aber auch auf größeren Technologie- und Wirtschaftsgebieten zu beobachten und etwa bestimmte Felder der Hochtechnologie zu definieren und zu analysieren. Auch lasse sich feststellen, welche Wirtschaftsbereiche die technische und wirtschaftliche Entwicklung tragen. Angestrebt wird „ein Gesamtbild, das die technologische Struktur und ihre Veränderungen widerspiegelt".

Der Vergleich der Herkunft der Patente mit ihrer Anwendung in den einzelnen Branchen zeigt zwar eine weitgehend gleiche Verteilung; an der Spitze stehen (in dieser Reihenfolge) Elektrotechnik, Maschinenbau, Chemische Industrie, Kraftfahrzeugbau sowie Feinmechanik und Optik. Eine weitere Aufschlüsselung macht aber eine je nach Wirtschaftszweig mehr oder weniger starke Streuung deutlich. Insgesamt sind in $67 \%$ der Fälle Herkunfts- und Verwendungsbranche identisch. In Feinmechanik/Optik kommen jedoch nur $30 \%$ der verwendeten Patente aus der Branche selbst, in der Chemie dagegen sind es $92 \%$, in der Elektrotechnik $79 \%$, im Maschinenbau $63 \%$ und im Kraftfahrzeugbau $56 \%$. Starke Variationen lassen sich auch feststellen bei einem Vergleich der Anteile der FuE-Ausgaben mit den Anteilen der Patentanmeldungen. Daraus ist zum Beispiel zu ersehen, daß im Maschinenbau relativ viele kleine Patente angemeldet werden, entsprechend der stark mittelständisch geprägten Branchenstruktur, in der Chemie dagegen relativ große. Die Konkordanz soll weiterentwickelt und verfeinert werden, so daß sie Zusammenhänge zwischen den FuE-, Erfindungs- und Innovationsaktivitäten auf der einen, Unternehmensgrößen, Produktion, Arbeitsproduktivität, Konjunktur und Wirtschaftswachstum, Investitionen, Marktgeschehen auf der anderen Seite aufzeigen kann.

Auch soll sie den internationalen Bereich mit einbeziehen, insbesondere die Aktivitäten der in der Bundesrepublik agierenden ausländischen Unternehmen. Auf diese Weise sei es möglich, „Aufschlüsse darüber zu erlangen, in welchen Bereichen und gegenüber welchen Ländern technologische Lücken und Abhängigkeiten bestehen".

\section{Zentren für Ökosystemforschung}

Die seit langem bekannten Unzulänglichkeiten der ökologischen Forschung sollen jetzt überwunden werden. Der ganzheitliche Ansatz der Ökosystemforschung, den das Bundesforschungsministerium künftig fördern will, soll ökologische Prozesse, Ursachen- und Wirkungszusammenhänge besser erklären, als dies mit isolierten Untersuchungen der 